\title{
La maille et le nœud : apprendre en réseau
}

\section{Joseph Rézeau}

\section{Q OpenEdition \\ Journals}

Édition électronique

URL : http://journals.openedition.org/asp/2625

DOI : 10.4000/asp.2625

ISBN : 978-2-8218-0380-0

ISSN : 2108-6354

Éditeur

Groupe d'étude et de recherche en anglais de spécialité

Édition imprimée

Date de publication : 1 décembre 1999

Pagination : 463-484

ISSN : 1246-8185

Référence électronique

Joseph Rézeau, «La maille et le nœud : apprendre en réseau », ASp [En ligne], 23-26 | 1999, mis en ligne le 18 janvier 2012, consulté le 20 avril 2019. URL : http://journals.openedition.org/asp/2625 : DOI : 10.4000/asp.2625

Ce document a été généré automatiquement le 20 avril 2019

Tous droits réservés 


\section{La maille et le noud : apprendre en réseau}

Joseph Rézeau

\section{Introduction}

1 Le concept de réseau, pour n'être pas nouveau, n'en est pas moins omniprésent dans notre société, avec l'importance considérable prise par l'Internet, le «réseau des réseaux $"$.

2 Réseau provient étymologiquement du latin retis (filet). À travers une longue filiation composée de rets, de résel, et de réseul (qui, au $16^{\mathrm{e}}$ siècle désignait... un soutien-gorge), on parvient à réseau (au $17^{\mathrm{e}}$ siècle). À partir du $18^{\mathrm{e}}$ siècle, la médecine s'empare métaphoriquement du réseau, d'abord pour décrire puis pour rendre compte du fonctionnement de l'organisme humain. Non seulement le corps est tissu, filet reliant différents éléments en un tout, mais il est aussi irrigué par un flux circulatoire. Puis, au 19 ${ }^{e}$ siècle, le concept de réseau s'appliquera successivement aux domaines de la poste, des routes et des chemins de fer, et de la distribution de l'eau; tous ces domaines venant renforcer l'aspect circulatoire du concept de réseau. Avec les réseaux télégraphiques et téléphoniques s'y ajoutera la notion de communication. À partir du milieu du $20^{\mathrm{e}}$ siècle, la notion d'information réinvestit la métaphore réticulaire, avec les réseaux de machines et d'automates d'une part et les théories des réseaux de neurones d'autre part ${ }^{1}$.

3 La puissance du concept de réseau, évoquée au fil de ce bref parcours étymologique, nous incite à examiner les applications métaphoriques de ses diverses acceptions dans le domaine de la didactique des langues.

4 Nous commencerons par la métaphore du filet, patiemment fabriqué et tendu par le pédagogue pour capturer l'intérêt des apprenants et faciliter leur propre 'pêche au savoir' ; ce sera notre premier point : prendre pour apprendre. Nous évoquerons dans un deuxième point le pouvoir organisationnel du réseau : comprendre pour apprendre. 
Pour conclure, nous tirerons de notre parcours des implications pratiques pour proposer un apprentissage de la langue en réseau.

\section{Le réseau comme filet : prendre pour apprendre}

\section{De la nécessité d'un filet}

Il tendit un long rets. Voilà les poissons pris [...]

(La Fontaine, Fables, X : 10)

5 À moins de travailler dans un cadre d'enseignement où il s'adresse à un auditoire captif, l'enseignant devra tout d'abord aller à la pêche ... aux enseignés. Et même un public théoriquement captif peut s'échapper du cadre éducatif institutionnel et «aller voir ailleurs [...] dans la cave d'un copain qui démonte sa mobylette» (Mérieu 1987:16) et ainsi grossir les effectifs des décrocheurs et décrocheuses, comme les appellent nos cousins canadiens (Bibeau 1998).

6 Le cadre de notre enseignement est celui que Monique Mombert présentait comme le «post-bac» ou «l'enseignement aux non-spécialistes» dans le numéro spécial de la revue des Langues Modernes consacré à ce thème. Si nous reprenons sa classification de ce type d'enseignement en deux pôles opposés, notre situation correspond au pôle négatif, caractérisé par les éléments suivants :

... étudiants non sélectionnés ou sélectionnés sur d'autres matières que les langues

[...] ; pas ou peu de valorisation des langues dans le cursus ; enseignants vacataires,

peu de moyens... (Mombert, 1993 : 10).

7 Dans un tel contexte, il devient vite évident que l'enseignant doit tendre un bien long rets pour capturer les apprenants, afin d'éviter qu'un trop grand nombre d'absentéistes ne passent au travers des mailles. Parmi l'attirail des ressources didactiques à la disposition des enseignants de langues, les technologies de l'information et de la communication, rebaptisées "multimédia » sont souvent présentées comme une panacée susceptible de motiver les apprenants les plus rétifs. Mais hormis l'évocation générale de «l'enthousiasme des apprenants» les preuves tangibles des vertus de ces nouvelles technologies sont rarement fournies, faute d'être mesurées ou mesurables. L'expérience que nous avons menée sur quatre années consécutives auprès d'une population d'étudiants de DEUG 1 Histoire de l'art montre l'impact significatif d'un environnement multimédia d'apprentissage tout au moins sur l'assiduité des sujets de l'expérience. La population concernée suivait alternativement un cours de travaux dirigés de type traditionnel (TD) une semaine, et un cours multimédia (MM) l'autre semaine. Le graphique de la Figure 1 montre le taux d'assiduité au cours d'anglais rapporté au nombre d'étudiants présents à l'examen de fin d'année selon le type de cours: TD ou MM. On constate d'une part que l'écart des taux d'assiduité est constamment en faveur du cours de type multimédia et d'autre part que l'écart est d'autant plus grand que l'assiduité globale est faible (années 1996 et 1999). 
Figure 1. Comparaison des taux d'assiduité selon le type de cours

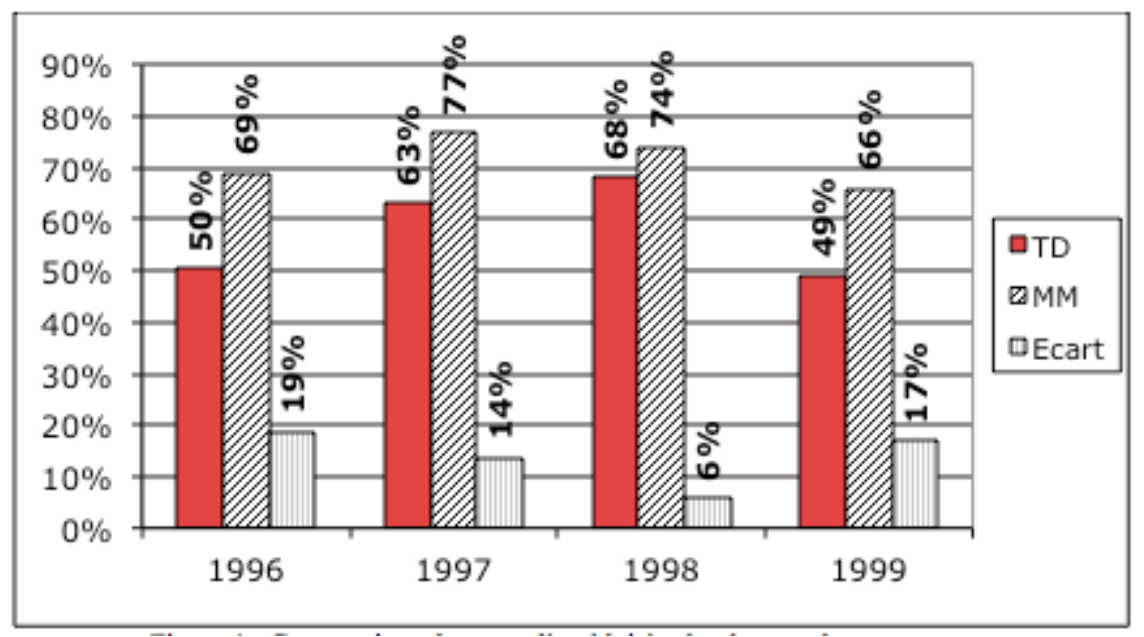

\section{De la transparence du filet}

Surely in vain the net is spread in the sight of the

bird.

(Proverbs I, 17)

8 Comme nous le rappelle l'auteur anonyme et inspiré du Livre des Proverbes, le filet sera d'autant plus efficace qu'il sera moins visible. Une technique efficace de 'camouflage' consiste à dissimuler les mailles dans le paysage environnant, par exemple en faisant de la langue de spécialité. Cette 'fusion' du filet dans le paysage ne constitue pas seulement un moyen (passif) de camouflage du dispositif didactique, elle fournit un élément supplémentaire (actif) de motivation à l'apprentissage.

Il arrive même qu'on assiste à une inversion des termes dans la perception des objectifs de ce type de cours par les apprenants, comme en témoigne la remarque suivante d'un étudiant qui place l'objectif de l'apprentissage de la langue en seconde position, après un apprentissage du contenu.

I think it is a very interesting way to learn art history. It is also a very interesting way to learn English...

10 Ce point de vue est d'ailleurs corroboré par les réponses à l'enquête de fin d'année, dans laquelle les étudiants doivent classer par ordre de préférence une dizaine de types de motivation offerts par les leçons de type multimédia (Figure 2). La motivation 'utilité pour l'étude de l'histoire de l'art' est toujours classée avant la motivation 'utilité pour l'étude de l'anglais'. L'écart dans le classement, très net de 1996 à 1998, se réduit à 1 en 1999, sans que les commentaires des étudiants apportent d'explication à ce phénomène. Tout au plus aimerions-nous penser que ce 'progrès' relatif de la perception de l'utilité du cours multimédia pour l'apprentissage de la langue résulte d'une amélioration du contenu didactique de nos leçons, sans cesse remaniées au fil de ces quatre années... 
Figure 2. Classement des motivations d'apprentissage dans un cours multimédia.

\begin{tabular}{|l|l|l|l|l|l|}
\hline motivations & 1996 & 1997 & 1998 & 1999 & $\begin{array}{l}\text { rang } \\
\text { moyen }\end{array}$ \\
\hline active learning & 1 & 1 & 1 & 2 & 1 \\
\hline autonomous work & 3 & 3 & 4 & 1 & 2 \\
\hline intellectually stimulating & 4 & 2 & 2 & 5 & 3 \\
\hline usefulness for Art studies & 6 & 4 & 3 & 3 & 4 \\
\hline aesthetic pleasure & 2 & 5 & 6 & 7 & 5 \\
\hline constructing our knowledge & 5 & 7 & 5 & 6 & 6 \\
\hline using modern technology & 7 & 6 & 8 & 8 & 7 \\
\hline usefulness for learning English & 9 & 9 & 7 & 4 & 8 \\
\hline etc. & & & & & \\
\hline nombre de réponses & $\mathbf{6 1}$ & $\mathbf{1 0 8}$ & $\mathbf{1 2 5}$ & $\mathbf{9 8}$ & \\
\hline
\end{tabular}

\section{De l'importance de l'appât}

Quand le pêcheur amorce l'eau, le poisson vient.

(Rousseau, Émile, IV).

11 Les théoriciens de la motivation nous disent que, pour provoquer celle-ci, il faut créer un déséquilibre entre une situation perçue comme non satisfaisante et une situation perçue comme satisfaisante. Si nous filons la métaphore de la pêche, le témoignage suivant nous permet d'illustrer l'intérêt de l'appât du matériau didactique pour attirer l'étudiantpoisson dans notre filet!

...using this program is wonderfull, $\mathrm{i}$ think historical context [art history] can be a good way to give interest to the horible monster cald passiv form; although i will never can be his master

Dans une pédagogie de la motivation, le rôle du pédagogue sera d'appâter l'apprenant, de créer ce déséquilibre entre ce qu'il possède et ce qu'il n'a pas, de poser un problème que l'apprenant aura envie de résoudre. C'est là, comme nous le rappelle M. Perrin :

[...] le principe méthodologique qui est à la racine de la plus grande partie de l'activité du formateur en langues: [...] créer du manque, installer de l'écart, produire du vide à partir du plein, de manière à ce que les apprenants soient mis devant la nécessité [...] de combler ces manques, réduire ces écarts, remplir ces vides. (Perrin 1999: 9)

13 Mais le pédagogue le plus inventif n'est-il pas désarmé face au manque de motivation (à l'amotivation) ? On ne fait pas boire un âne... qui n'est pas motivé, comme nous le rappelle 
Jean Cureau, dans le numéro spécial des Langues modernes sur la motivation (Cureau, 1985). On donne au phénomène de l'amotivation les causes suivantes: l'apprenant s'estime incompétent ou bien il ne se donne pas de buts ou encore il ne fait pas de lien entre son comportement et les conséquences de son comportement. Voici quelques illustrations de ces trois motifs d'amotivation dans notre population étudiante.

L'estimation d'incompétence (associée ici à une lucide reconnaissance de responsabilité).

I begin to see my main weakness : I don't know my english grammar!!! of course, that's not really something new, I knew it before I came here. (Nobody's fault but mine, I've never worked my English lessons in the past.)

Voici une déclaration bien paradoxale où la forme dément le fond. Que peut bien vouloir démontrer un étudiant qui déplore son ignorance de la grammaire anglaise en s'exprimant avec une syntaxe parfaite? Ne sommes-nous pas en présence d'un cas typique d'évocation de son incompétence par l'apprenant, comme excuse pour refuser la tâche d'apprentissage à accomplir, qu'il déclare a priori infaisable? Cette tendance est mise en avant par J.C. Vignaud en introduction à son article sur le « progrès » en langues :

Placé dans des conditions peu favorables à l'apprentissage d'une langue vivante, évalué à l'aune du natif, l'élève a tendance à évoquer ses incompétences [...] plutôt que ses compétences. (Vignaud $1997: 24)$

Le manque de but : à leur arrivée à l'université, un bon nombre d'étudiants de notre population n'ont aucun projet précis concernant leur avenir après les études. L'enquête que nous menons systématiquement en début d'année révèle en effet qu'ils étaient $23 \%$ dans ce cas en 1994-95 et $32 \%$ en 1998-99. Or les praticiens comme les théoriciens de l'apprentissage insistent sur le fait qu'il ne saurait y avoir apprentissage s'il n'y a pas de projet d'apprendre.

Le manque de lien entre un comportement donné et les conséquences de ce comportement conduit souvent à agir au hasard. Comme le souligne Porcher, un grand nombre d'apprenants se trouvent dans une situation où,

statistiquement, les chances d'échec sont plus nombreuses que les chances de succès. En termes de probabilité, [...] ils ont intérêt à se laisser aller au hasard.

(Porcher 1985 : 107)

En voici une illustration, extraite d'un dialogue entre deux étudiants travaillant sur un exercice de QCM d'une leçon multimédia :

B ouais... j'sais pas, au hasard, vas-y, dis un nom

A çui-là, y m'paraît bien, tiens

Une autre encore, sur un exercice de type lacunaire avec des quantificateurs :

B et si je mets $a$ few ?...

A on peut mettre a few...

B vas-y, essaie a few...

A y a que $a$ few, de toute façon

B ben oui, le reste... donc voilà... merde ! c'est pas celui-là !.. 


\section{De l'importance de la rétention}

...while memory holds a seat in this distracted

$$
\text { globe... }
$$

(Hamlet, I, v, 96) l'apprentissage distribué sur l'apprentissage massé :

I learnt new vocabulary, espcially technical terms of painting. The fact that they are used several times in the lesson and in different sentences help me to memories them.

De nombreuses remarques de nos étudiants concernent l'effet de 'rafraîchissement de la mémoire' (que nous attribuerons dans notre prochain point au renforcement des connexions) procuré par les rappels grammaticaux des leçons multimédias.

And more, this lesson on comparatives and superlatives was a good one in the sense that after two months of vacancy I didn't remember of all.

I had forgotten the quantifiers and it's interresting to see them again.

I had forgotten what was the passive voice and now I remember all my old lessons!

I have learnt this painting many days ago, because I'm going to pass a exam about the paleolithic, so it's good for me to work this painting because it helps me to remember a lot of things that I shoud know, in order to manage my exam.

21 Un stimulus (en particulier un stimulus de type visuel) peut avoir un impact significatif sur la mémorisation d'un item :

This painting is very beautiful and very interesting. I will remember her!

Pour conclure ces considérations sur la mémorisation, le temps est considéré par nos étudiants comme le grand responsable de l'oubli :

In fact, the time is our enemy (we forget!)

La métaphore du réseau comme filet nous a permis de mettre en évidence l'importance des outils didactiques dans la capture de l'intérêt des apprenants. Mais cette métaphore se révèle insuffisante pour rendre compte des phénomènes de rétention et de mise en œuvre des connaissances. Il nous faut donc poursuivre notre voyage étymo-métaphorique et passer de la métaphore du réseau comme filet servant à capturer l'information à celle 
du réseau permettant d'organiser et donc de stocker l'information dans sa propre structure : passer de l'outil-capteur à l'outil-support.

\title{
Le réseau comme connecteur : comprendre pour apprendre
}

\author{
Where is the Life we have lost in living? \\ Where is the wisdom we have lost in knowledge? \\ Where is the knowledge we have lost in \\ information? \\ (TS Eliot Choruses from The Rock, I)
}

Dans la métaphore du réseau comme filet, le filet sert seulement à capturer l'information, il ne la véhicule pas. Dans cet usage, on considère l'apprentissage comme un processus d'acquisition de connaissances et d'information. Mais apprendre ce n'est pas seulement s'informer, ou plutôt il convient de considérer l'information comme le niveau zéro de l'apprentissage.

D'ailleurs, peut-on dire qu'on apprend mieux ou plus dans la société contemporaine, où nous sommes sur-informés? Ce problème de la saturation d'information était déjà évoqué par Reboul il y a près de 20 ans :

Le (télé)spectateur n'apprend pas parce qu'il apprend trop... par la satisfaction qu'elle donne, par l'illusion de savoir qu'elle procure, l'information empêche

d'apprendre. (Reboul $1980: 24$ )

Ce phénomène est encore amplifié avec l'accès à Internet, où la saturation d'information atteint un niveau quasi illimité. Puisque nous évoquons ici le concept d'information, il nous paraît utile de préciser les rapports entre ce concept et celui de communication, qui lui est souvent associé, et ceci dans le cadre des réseaux.

\section{Réseaux d'information vs. réseaux de communication}

Les auteurs contemporains s'accordent à distinguer les réseaux véhiculant l'information de ceux véhiculant la communication selon les deux systèmes présentés dans le tableau de la Figure 4.

Figure 3. Réseaux d'information et de communication

\begin{tabular}{|c|c|c|c|}
\hline & INFORMATION & \multicolumn{2}{|l|}{ COMMUNICATION } \\
\hline $\begin{array}{l}\text { fonction } \quad \mathrm{du} \\
\text { réseau }\end{array}$ & distribution & connexion & \\
\hline exemples & télévision, radio & $\begin{array}{l}\text { téléphone, Internet, } \\
\text { d'ordinateurs }\end{array}$ & réseaux \\
\hline type d'organisation & hiérarchique & $\begin{array}{l}\text { non hiérarchisée, } \\
\text { démocratique }\end{array}$ & collective, \\
\hline
\end{tabular}




\begin{tabular}{|c|c|c|}
\hline $\begin{array}{ll}\text { sens } & \mathrm{du} \\
\text { déplacement } & \end{array}$ & top 䃈 down & inter niveaux \\
\hline $\begin{array}{l}\text { problèmes de } \\
\text { coupure }\end{array}$ & $\begin{array}{l}\text { grave : l'information ne passe } \\
\text { plus du tout }\end{array}$ & $\begin{array}{l}\text { sans importance: il suffit d'emprunter } \\
\text { un autre chemin }\end{array}$ \\
\hline type de traitement & linéaire, séquentiel & (massivement) parallèle \\
\hline $\begin{array}{ll}\text { activité } & \text { de } \\
\text { l'utilisateur } & \end{array}$ & passif & actif \\
\hline enseignement $^{3}$ & la didactique & la pédagogie \\
\hline
\end{tabular}

Cette opposition entre les deux types de réseaux est ainsi mise en évidence par Negroponte :

A television network is a distribution hierarchy with a source (where the signal comes from) and multiple heterogeneous sinks (where the signals go to). Computer networks, on the other hand, are a lattice of heterogeneous processors, any one of which can act both as source and sink. (1995:180)

Joël De Rosnay, dans Le macroscope, parle de deux systèmes pour l'information, l'un pour l'information descendante (type distribution) et l'autre pour l'information montante.

Ces deux systèmes sont le prolongement, au niveau social, des deux modes d'action fondamentaux de la conscience individuelle : l'observation (en vue d'acquérir des connaissances, de s'informer) et l'action créatrice (en vue d'organiser le monde , d'informer la matière). (1975: 200)

Il ajoute que, de ces deux modes d'action, le premier est considéré comme le plus facile, et le second comme le plus astreignant. Ceci se vérifie quotidiennement, tant au niveau individuel qu'au niveau social. Dans une visée thermo-dynamique de la théorie de l'information, toute action d'acquisition de connaissances consomme de l'information et donc augmente l'entropie de l'univers, tandis que toute action créatrice est au contraire néguentropique, et ajoute de l'information à la matière ${ }^{4}$.

La différence de difficulté perçue entre les deux modes d'action de l'individu : acquisition d'information d'une part et organisation (de ces informations) d'autre part peut être illustrée par un certain nombre de remarques de notre public habituel.

Cet étudiant fait preuve de lucidité sur son manque d'organisation dans ses études, et les conséquences que cela entraîne quant à son incapacité à progresser :

As $i$ don $t$ looking for a organisation in my english study (or else), $i$ can $t$ make progres.

Dans nos leçons multimédias, nous proposons une activité de remise en ordre des mots ou groupes de mots d'une phrase anglaise. Les étudiants sont unanimes à déclarer qu'il n'est pas facile de «créer de l'ordre » et pourtant ils peuvent passer un temps relativement long à s'y essayer, sans se lasser. Une activité créatrice d'ordre (néguentropique) seraitelle donc motivante? 


\section{Les réseaux neuronaux} code.

ns L'homme neuronal, Changeux décrit le cerveau comme un réseau de réseaux ${ }^{5}$ :

L'encéphale de l'homme se présente à nous comme un gigantesque assemblage de dizaines de milliards de «toiles d'araignées » neuronales enchevêtrées les unes aux autres [...]. (1983:160)

Dans le contexte des sciences cognitives, un réseau neuronal artificiel est un modèle d'automate formel conçu sur l'analogie des réseaux neuronaux du système nerveux pour modéliser le fonctionnement du cerveau humain et notamment des processus d'apprentissage. Les « réseaux neuronaux » ont la particularité de sécréter de l'ordre et, s'ils sont correctement conçus, ils ont également la capacité d'apprendre. (Voir Raynal, 1997 : 323). Comme nous l'avons indiqué plus haut (Figure 4) un gros avantage d'un réseau dont la fonction est la connexion plutôt que la distribution est sa relative insensibilité aux problèmes de coupure.

Il faut toutefois préciser que l'analogie n'est pas totale entre le fonctionnement d'un réseau interconnecté, de type Internet, d'une part et celui d'un réseau neuronal :

- Dans le cas du réseau interconnecté, l'information située en un point précis est en principe toujours accessible même en cas de coupure du réseau en un ou plusieurs points ; il suffit d'emprunter un autre chemin. On sait que c'est précisément cet avantage de continuité de l'information qui a été à l'origine historique du réseau ARPANET créé par le ministère américain de la défense en 1968.

- En ce qui concerne le réseau neuronal, c'est l'information elle-même qui peut être obtenue à partir de différentes configurations du réseau, par compensation de poids synaptiques. En effet, le réseau neuronal n'est pas seulement le véhicule de l'information, il en constitue le

Cette caractéristique de relative fiabilité des réseaux fait des réseaux sémantiques la représentation privilégiée de la mémoire à long terme.

\section{Les réseaux sémantiques}

L'un des premiers modèles de mémoire à long terme dans lequel les réseaux associatifs sémantiques jouent un rôle important est le Teachable Language Comprehender (TLC) présenté par Ross Quillian. L'organisation du TLC est de type hiérarchique, en arbre, chaque nœud représentant les concepts et les traits symbolisant la relation d'inclusion entre concepts. (Cité par Weil-Barais $1993:$ 389).

Toutefois, le principe d'économie cognitive de ce modèle ne se vérifie pas toujours, et d'autres modèles, non hiérarchiques, ont été proposés, comme les "mind maps ». Un avatar tout récent et 'didactisé' de ces cartes conceptuelles est l'activité de création de Word Webs proposée dans le manuel Wordflo (édité par Longman). Selon les auteurs, le but de cette activité est «d'aider les étudiants à mémoriser le vocabulaire nouveau en les encourageant à l'organiser sous une forme visuellement intéressante ».

Pour Lévy (1990: 92), «la compréhension est l'association d'un item d'information avec un schéma préétabli». Cette association est également un moyen de stocker l'information en mémoire à long terme, en vue d'une réactivation ultérieure.

Selon Mislevy (1990) rapporté par De Landshere (1992 :57) : 
[...] comparés aux novices, les experts dominent plus de faits et établissent plus d'interconnexions ou de relations entre eux. Ces interconnexions permettent de surmonter les limitations de la mémoire à court terme. Alors que le novice ne peut travailler qu'avec au maximum sept éléments simples, l'expert travaille avec sept constellations incarnant une multitude de relations entre de nombreux éléments.

$\mathrm{Au}$ terme de ce parcours métaphorique, nous voyons que l'intérêt principal de l'application de la métaphore réticulaire au domaine de l'apprentissage est de mettre l'accent sur l'importance de la connectivité. Encore faut-il que les apprenants établissent de 'bonnes connections'.

\section{‘Only connect?’}

Only connect! That was the whole of her sermon.

Only connect the prose and the passion, and both

will be exalted,

and human love will be seen at its height.

(E.M. Forster, Howards End, XXII.)

Dans cette troisième et dernière partie de notre exposé, nous allons illustrer les réseaux conceptuels à l'œuvre 'en ligne' (pour rester dans le vocabulaire du réseau des réseaux) chez nos apprenants. Nous observerons tout d'abord des situations d'associations d'idées spontanées, puis nous ferons part d'une brève expérimentation de 'carte conceptuelle' provoquée.

\section{Associations d'idées et réseaux conceptuels spontanés}

L'art... alimentaire

43 La tâche proposée à des étudiants en histoire de l'art consiste à compléter avec un adjectif composé la phrase : « a cake which is not bought from a shop is ... a cake»

B un gâteau, un gâteau qui n'a pas été acheté dans une...

A ben, c'est fait à la maison...

B fait-main

A non, made home?

B non, c'est home-made

A essaie home-made

B oui... ready-made ?

A non, ça c'est Duchamp, ready-made !

B ah ben, on est en histoire de l'art, hein !

La publicité plus forte que l'art
A dove, c'est pigeon?
B oui, dove, tu sais j'ai retenu avec le...
A Piero della Francesca, non?
B non mais avec le savon, aussi, dove

On observe dans ces deux premiers cas que le réseau conceptuel de la spécialité des étudiants (histoire de l'art) tantôt s'impose tantôt laisse la préséance à la publicité omniprésente dans la vie quotidienne des sujets.

\section{En avoir ou pas}


B non, ox c'est ça: (B lit une définition à l'écran) a fully grown male of the cattle family, with sexual organs removed

A c'est quoi removed?

B heu, à mon avis, attends, heu, il s'est fait, heu, ils sont castrés les bœufs

A je sais pas

B ben, je crois, parce que autrement, ce serait des taureaux

A je sais pas, je vois pas la différence

...

A heu, c'est un taureau, je sais pas comment on dit un taureau... une vache, déjà, comment on dit une vache?

B a cow!

A a cow, cow-boy!... de l'apprenant. Il est en effet bien difficile pour A de raccrocher un concept de castration à l'absence de réseau conceptuel vache / bœuf / taureau. En revanche, grâce au réseau bien implanté dans les mémoires par les Westerns et autres Lucky Luke, cow est solidement accroché à cow-boy. On retrouve ici le problème fondamental du rôle de la culture générale dans l'apprentissage. C'est un lieu commun pédagogique que de déplorer le manque de culture générale de nos étudiants à leur arrivée à l'université. S'il est une bonne raison de regretter ce déficit, ce n'est pas au nom d'un élitisme qui conduirait à refuser l'accès à ce type d'institution à ceux qui présenteraient cette tare. Il s'agit bien, en revanche, d'attirer l'attention de nos étudiants sur le fait que l'apprentissage est un tout, et que, pour être d'abord compris puis efficacement mémorisé, un item nouveau doit être associé, raccroché, interconnecté à un schéma préétabli, à un réseau préexistant dans la culture générale de l'apprenant.

\section{Associations provoquées}

Afin de mesurer l'influence de la situation d'apprentissage sur la réorganisation des réseaux conceptuels, nous avons organisé l'expérience suivante. Juste avant une leçon multimédia sur le thème du Guernica de Picasso, nous avons demandé à 42 étudiants de disposer librement sur une page d'écran 24 mots proposés (il s'agissait de mots qu'ils allaient rencontrer ensuite dans la leçon mais cette information ne leur était pas communiquée). Cette première activité a constitué une sorte de "pré-test ». Aussitôt après la leçon, la même activité a été proposée aux sujets, avec les mêmes mots (le "posttest »), et les deux versions successives de leurs 'word webs' ont été sauvegardées pour être étudiées.

\section{L'intégration des mots nouveaux}

En accord avec la consigne donnée, les mots qui sont restés à leur place d'origine sont soit des mots inconnus des étudiants (tels que gash, rag, sword, etc.), soit des mots reconnus mais que les étudiants ne savaient pas comment regrouper avec les mots connus (exemple probable : broken).

Figure 4. Mots non intégrés à des réseaux sémantiques

\begin{tabular}{|l|l|l|l|} 
mots non utilisés & avant & après & Hypertexte
\end{tabular}




\begin{tabular}{|l|l|l|l|}
\hline gash & 35 & 6 & $\mathrm{x}$ \\
\hline sword & 32 & 11 & \\
\hline limp & 30 & 3 & $\mathrm{x}$ \\
\hline rag & 30 & 13 & \\
\hline droop & 27 & 5 & $\mathrm{x}$ \\
\hline broken & 20 & 4 & \\
\hline foreshorten & 17 & 1 & $\mathrm{x}$ \\
\hline bull & 12 & 3 & \\
\hline Harlequin & 9 & 1 & $\mathrm{x}$ \\
\hline horse & 6 & 0 & \\
\hline doll & 1 & 2 & \\
\hline hope & 5.6 & $\mathbf{1 . 7}$ & \\
\hline bottom & 5 & 5 & \\
\hline magical & 2 & 2 & \\
\hline foreground & 1 & 0 & \\
\hline mystical & 1 & 0 & \\
\hline perspective & 3 & \\
\hline moyenne & 5 & \\
\hline
\end{tabular}

On voit que, dans les réseaux constitués à l'issue de la leçon multimédia d'anglais sur le thème Guernica, le nombre de mots encore 'inconnus' ou insuffisamment connus par les étudiants pour être inclus dans des réseaux sémantiques a considérablement diminué par rapport au pré-test (Figure 6, colonnes 'avant' et 'après'). On constate également que, parmi les mots supposés inconnus avant la leçon, ceux qui sont le plus souvent utilisés en réseau après celle-ci étaient munis d'un lien hypertexte explicatif dans la leçon étudiée (ces mots sont marqués $\mathbf{x}$ dans la colonne hypertexte). En revanche, les mots sword et rag, non expliqués dans la leçon, continuent à poser problème pour environ un tiers des sujets qui ne les avaient pas intégrés à un réseau sémantique lors du pré-test et qui laissent encore de côté ces mots lors du post-test.

\section{Le novice, l'expert et la connectique}




\section{Le novice, l'expert et la connectique}

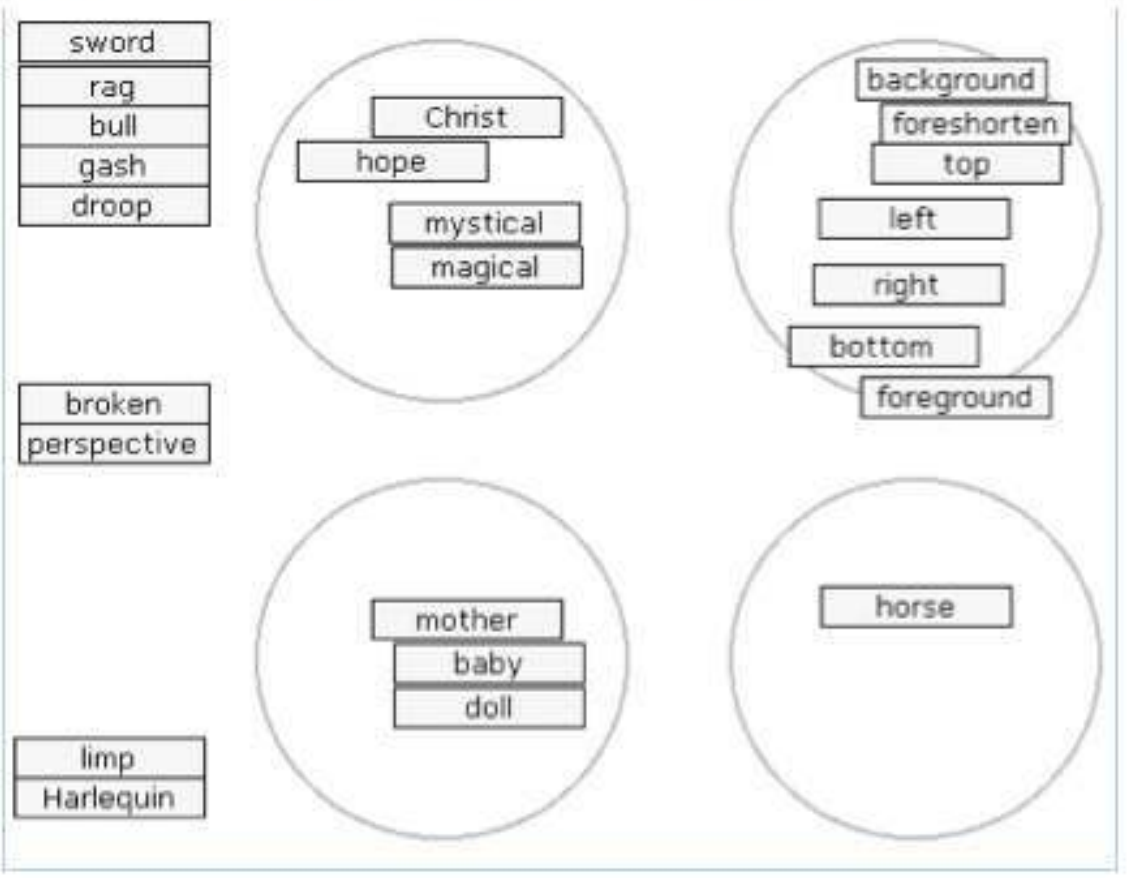

49 La figure 5 montre les réseaux sémantiques produits par un sujet de niveau faible (ayant obtenu moins de 6/20 à l'examen de fin d'année). On constate un nombre de mots non utilisés (9) plus élevé que la moyenne $(5,6)$, ce qui amène un 'réseau' ne comportant qu'un seul mot, horse. En revanche, il est surprenant de voir foreshorten placé à bon escient dans le réseau des concepts de localisation, alors que ce mot est inconnu de $40 \%$ des étudiants. 
Figure 6. Réseaux de mots produits par l'étudiant B au pré-test

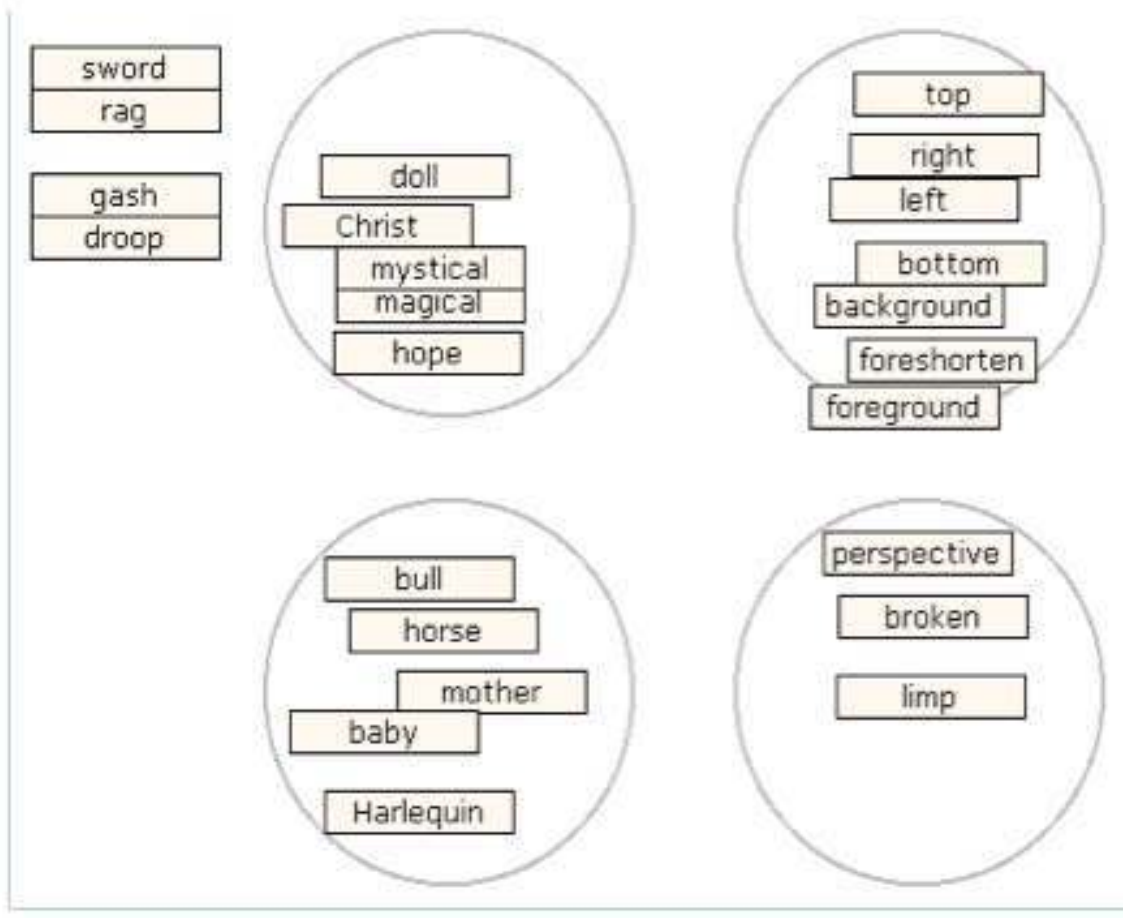

Après la leçon, le sujet A ne sait (ou ne veut) toujours pas placer 4 mots (contre 1,7 mots en moyenne pour le reste du groupe). Le réseau des concepts de localisation (quadrant supérieur droit) reste inchangé par rapport au pré-test, et les trois autres réseaux ne sont ni très convaincants ni en rapport avec la présentation des mots dans la leçon.

\section{Figure 7. Réseaux de mots produits par l'étudiant B au post-test}

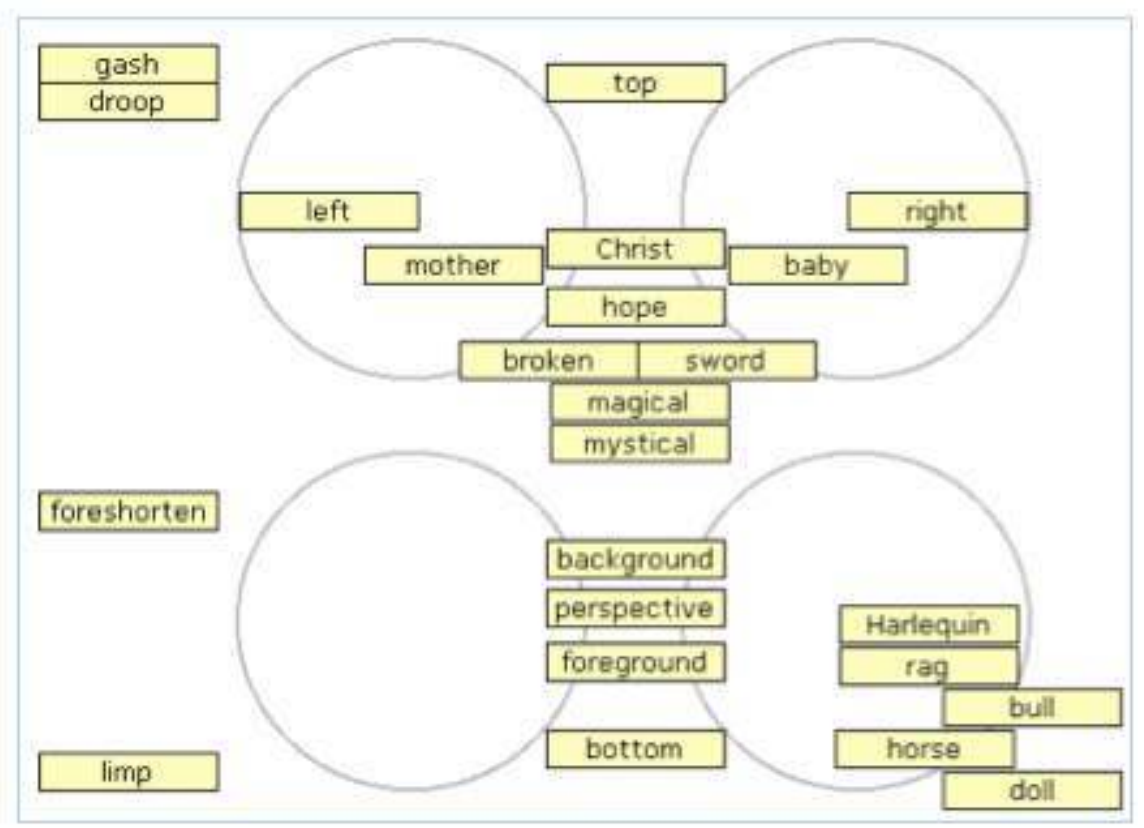


51 En revanche, la comparaison du pré-test et du post-test de l'étudiant B fournit un bel exemple d'expertise. (Il s'agit d'un étudiant qui a obtenu 14.5/20 à l'examen de fin d'année). Dans le réseau de mots du pré-test, cet étudiant ignore, comme la plupart de ses camarades, les mots gash, droop, foreshorten et limp. Mais il organise les mots qu'il connaît d'une manière très méthodique : au centre un réseau hiérarchique et spatial ; en bas à droite une sorte de 'réserve' de mots en attente.

Figure 8. Réseaux de mots produits par l'étudiant B au pré-test

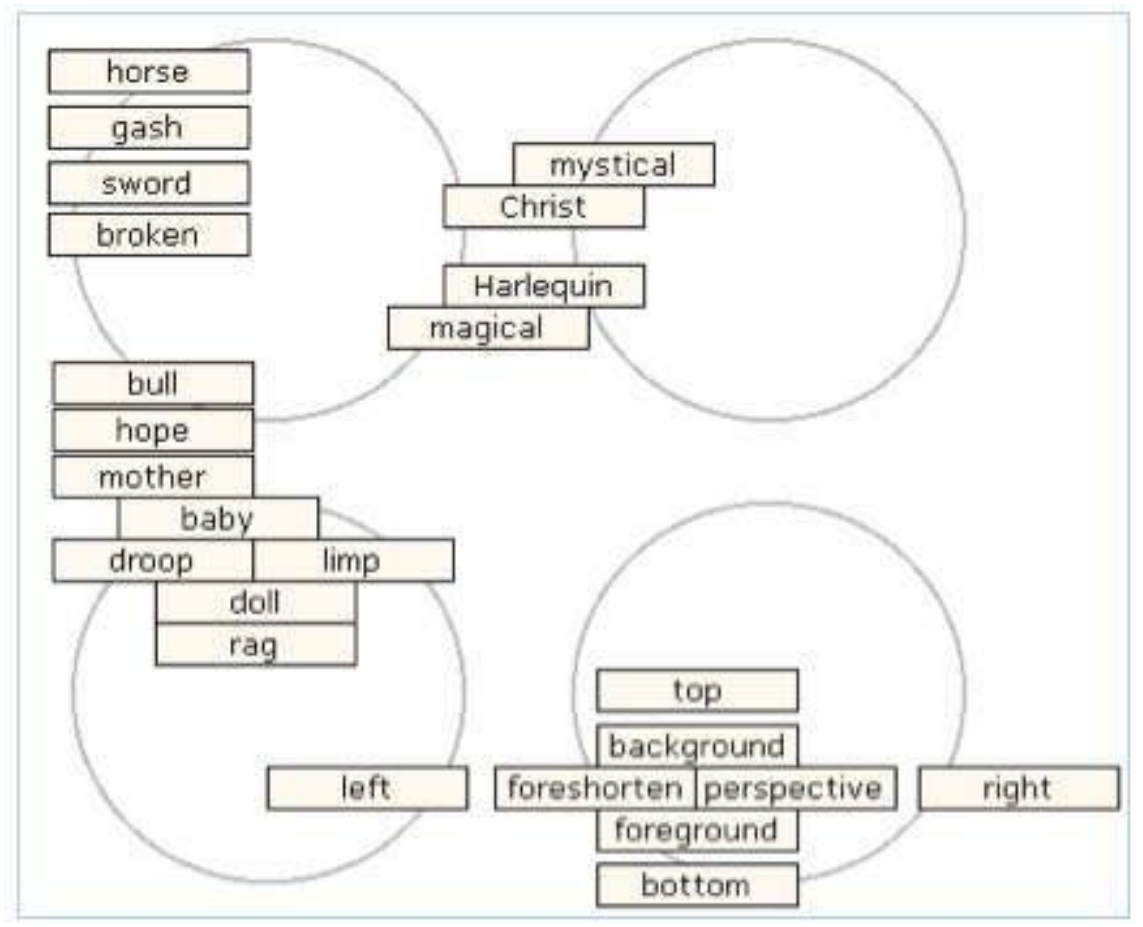

52 Au Post-test, l'étudiant B a parfaitement intégré les mots nouveaux, il n'en reste pas un seul d'inutilisé. Mais le plus remarquable est la réorganisation de ses réseaux sémantiques. On voit tout d'abord en bas un réseau des concepts de représentations spatiales, lui-même organisé de manière spatiale, ce qui semble logique mais que très peu d'étudiants ont réalisé. Les trois autres réseaux sémantiques sont disposés de telle manière que l'on pourrait pratiquement les superposer au Guernica de Picasso (moyennant de légers décalages). Ceci est d'autant plus remarquable que les étudiants n'avaient plus le tableau sous les yeux à l'écran lorsqu'ils étaient en train de travailler sur leur post-test... L'étude détaillée du fichier de suivi informatique enregistré pendant la leçon confirme par ailleurs la manière méthodique de travailler de l'étudiant $\mathbf{B}$ : exploration systématique de toutes les réponses possibles aux exercices de QCM, écoute dans l'ordre de toutes les phrases enregistrées, etc.

\section{De l'influence de l'apprentissage sur la réorganisation des réseaux sémantiques}

Le dispositif utilisé pour notre expérience permettait d'enregistrer, pour chaque réseau sémantique produit à l'écran par les sujets, les coordonnées spatiales de chaque mot lors du pré-test puis lors du post-test. Les données ainsi recueillies nous ont ainsi permis 
d'étudier, pour l'ensemble de la population de l'expérience (42 sujets), les caractéristiques de rapprochement ou d'éloignement de chacun des 24 mots par rapport à chacun des 23 autres entre le pré-test et le post-test. L'étude du coefficient de rapprochement/ éloignement de certains mots clés permet de mettre en évidence l'influence du contenu de la leçon sur la réorganisation des réseaux sémantiques des apprenants. Dans les tableaux qui suivent, on a indiqué le pourcentage du nombre des sujets qui ont rapproché les mots situés en tête de colonne du mot vedette. Un pourcentage plus ou moins égal à 50 \% indique qu'il n'y a eu en moyenne ni rapprochement ni éloignement des mots; un pourcentage inférieur à $50 \%$ indique un éloignement et un pourcentage supérieur à $50 \%$ indique un rapprochement. Ainsi, le maximum de rapprochement, noté par un pourcentage de $100 \%$, indiquerait que les deux mots auraient été rapprochés par $100 \%$ des sujets.

\section{L'Arlequin, la poupée et le Christ}

Figure 9

\begin{tabular}{|l|l|l|l|l|l|}
\hline Harlequin & Christ & mystical & magical & baby & doll \\
\hline & $81 \%$ & $81 \%$ & $76 \%$ & $50 \%$ & $43 \%$ \\
\hline
\end{tabular}

Confrontés dans le pré-test au mot Harlequin, les sujets n'ont eu aucun mal à reconnaître dans ce mot transparent son équivalent français Arlequin (cf. figure 9). Dans les réseaux sémantiques disponibles dans leur mémoire à long terme, ils le trouvent associé à une image de divertissement, peut-être de marionnette, et donc le placent près de doll et de baby.

Mais l'un des textes descriptifs accompagnant le tableau étudié dans la leçon fait état d'un tout autre type d'association:

Picasso identified with Harlequin whom he associated with Christ because of his mystical power over death. In Guernica, the artist invoked a number of normally unseen Harlequins to magically overcome the forces of death represented in the painting.

En conséquence, dans le post-test, une majorité d'étudiants rapprochent le mot Harlequin des trois mots évoqués dans ce texte: Christ, mystical et magical. Dans le tableau, on ne note pas de changement global pour l'association Harlequin-baby, mais une légère régression de l'association Harlequin-doll.

\section{L'entaille dans le flanc du cheval}

Comme on l'a vu plus haut (Figure 6), le terme gash vient en tête des mots inconnus de la majorité des sujets, et s'est donc trouvé non intégré à un réseau sémantique lors du prétest par 35 d'entre eux (sur 42).

Selon certaines interprétations, le Guernica de Picasso serait une ré-interprétation de la crucifixion, et le cheval au centre du tableau, avec sa profonde entaille dans le flanc, représenterait le Christ. Cette interprétation n'était pas documentée dans les textes de la 
leçon multimédia objet de l'expérience, et le terme gash apparaissait seulement dans le texte suivant :

In the centre there is a horse with a terrible gash in its side. Beneath the horse's feet lies a warrior, his eyes dislocated in death; one hand holds a broken sword and a flower, while the other is extended helplessly empty.

Dans ce texte, le mot gash était muni d'un lien hypertexte renvoyant à la définition suivante : gash = a large deep cut. En outre, le passage du curseur sur ce mot lors des déplacements de la souris faisait apparaitre sur la reproduction du tableau à l'écran un encadrement qui mettait en évidence l'entaille dans le flanc du cheval. Cependant, seulement 13 étudiants sur 42 ont sollicité l'aide hypertextuelle en cliquant sur le mot gash (voir figure 10).

Figure 10

\begin{tabular}{|l|l|l|l|}
\hline gash & horse & Christ & sword \\
\hline & $86 \%$ & $74 \%$ & $45 \%$ \\
\hline
\end{tabular}

L'examen de l'évolution des distances entre le terme gash et certains des autres mots à mettre en réseau qui s'est produite entre le pré-test et le post-test, permet les observations suivantes. Nous constatons tout d'abord que le contexte immédiat d'apparition du terme gash a conduit une proportion significative des sujets à le rapprocher de horse. En revanche, le mot sword, qui aurait pourtant pu être perçu comme l'instrument de cette blessure, n'a pas davantage été associé à gash dans le post-test, puisque sa distance entre les deux mots a au contraire augmenté chez $55 \%$ des sujets. Mais il est vrai que le mot sword reste non intégré à un réseau sémantique par le quart des sujets au post-test. Enfin, la distance entre gash et Christ s'est réduite chez $74 \%$ des sujets, ce qui semblerait indiquer une association conforme à l'interprétation de Guernica indiquée plus haut.

\section{Conclusion}

61 Il semble bien que les exemples étudiés dans ce troisième point corroborent l'idée de l'importance des réseaux conceptuels sémantiques dans la mémorisation et l'apprentissage. Les deux exemples du 'bon apprenant' et du 'mauvais apprenant' (l'expert et le novice) montrent que les apprenants qui ont le plus de difficulté à apprendre sont ceux qui ont le plus de mal à organiser les éléments appris en réseaux conceptuels. L'étude statistique de l'évolution des réseaux sémantiques avant et après une séquence d'apprentissage indique l'influence de l'apprentissage sur la réorganisation de ces réseaux.

\section{Conclusion générale}

62 Nous voici arrivé au terme de notre voyage étymo-métaphorique au pays des réseaux. En ce qui concerne notre domaine d'intérêt, la langue de spécialité, nous soulignerons l'intérêt de la motivation pour la capture de l'intérêt de l'apprenant ainsi que les 
avantages inhérents à la constitution de mind maps pour favoriser un apprentissage optimal.

Dans une perspective d'auto-apprentissage guidé, il est très utile de montrer aux apprenants comment constituer leur apprentissage en réseau, ainsi que l'explique fort bien J. Walski dans son article « Du guidage en autonomie guidée » dans le numéro spécial des Langues modernes consacré à l'autonomie :

afin que l'apprenant ne considère jamais un exercice donné comme une fin en soi, [...] il importe de constituer, autour de chaque exercice initial, un réseau d'exercices complémentaires, dits d'approfondissement (Walski 1993 : 64)

64 C'est sur le modèle proposé par J. Walski que nous proposons systématiquement une rubrique Where to go from here dans les fiches de travail rédigées pour nos étudiants travaillant en espace-langues. Malheureusement, une enquête de fin d'année auprès de notre public montre que moins de $20 \%$ des intéressés effectuent ce parcours complémentaire, et encore seulement 'de temps en temps'.

Seule une prise de conscience par les enseignants de l'impérieuse nécessité d'apprendre aux étudiants à se construire un apprentissage en réseau permettra, peut-être, à ceux-ci d'acquérir autre chose qu'un "savoir mosaique, fait de fragments décontextualisés" (Guillaume 1999: 69). Si donc nous sommes d'accord pour dire qu'apprendre c'est établir des connexions, il s'ensuit que l'un des rôles essentiels de l'enseignant sera de former ses étudiants à la fonction de commutation, fonction autour de laquelle Marc Guillaume a construit son essai L'empire des réseaux.

\section{BIBLIOGRAPHIE}

Bailly, Danielle. 1998. Les mots de la didactique des langues. Le cas de l'anglais. Lexique. Paris : Ophrys. Bateson, Gregory. 1964. Vers une écologie de l'esprit, tome I. Paris : Seuil.

Bibeau, Robert. 1998. « L'élève rapaillé ». CRAP, 20-21.

Carré, Philippe, André Moisan et Daniel Poisson. 1997. L'autoformation : psychopédagogie, ingéniérie, sociologie. Paris : Presses universitaires de France.

Changeux, Jean-Pierre. 1983. L'homme neuronal. Paris : Fayard, Coll. Pluriel.

Cureau, Jean. 1985. « On ne fait pas boire un âne... qui n'est pas motivé ! ». Les langues modernes 5, $29-40$.

Dieuzeide, Henri. 1994. Les nouvelles technologies. Outils d'enseignement. Paris : Nathan.

Encyclopcedia Universalis. 1995. Art. « Réseaux (philosophie de l'organisation)».

Gaonac’h, Daniel. 1995. « La mémoire dans l'apprentissage des langues vivantes ». Les langues modernes 2.

Guillaume, Marc. 1999. L'empire des réseaux. Paris : Descartes \& Compagnie.

Laborit, Henri. 1974. La nouvelle grille. Paris : Robert Laffont. 
Latour, Bruno. 1995 [1989]. La science en action. Paris : Gallimard ( $1^{\text {re }}$ éd. Éditions La Découverte).

Latour, Bruno. 1996. «Ces réseaux que la raison ignore - laboratoires, bibliothèques, collections ». In Christian Jacob et Marc Baratin (dir.), Le pouvoir des bibliothèques. La mémoire des livres dans la culture occidentale. Paris : Albin Michel, 23-46.

Landsheere, Vivianne De. 1992. L'éducation et la formation. Paris : Presses universitaires de France.

Lévy, Pierre. 1990. Les technologies de l'intelligence : l'avenir de la pensée à l'ère informatique. Paris : Éditions La Découverte.

Mérieu, Philippe. 1987. Apprendre... oui, mais comment. Paris : ESF.

Mombert, Monique. 1993. « Le 'Post-bac' ou l'enseignement aux 'non-spécialistes’ « . Les langues modernes 2, 9-19.

Oudart, Pierre (dir.). 1997. « Multimédia, réseaux et formation », Le français dans le monde, Recherches et applications, Numéro spécial juillet.

Perrin, Michel. 1999. « Le plus vieux métier du monde n'est pas celui qu'on croit ». La Tribune Internationale des Langues Vivantes 25, 5-15.

Raynal, Françoise et Alain Rieunier. 1997. Pédagogie : dictionnaire des concepts clés. Paris : ESF.

Reboul, Olivier. 1980. Qu'est-ce qu'apprendre? Paris : Presses universitaires de France.

Rosnay, Joël De. 1975. Le macroscope. Paris : Seuil.

Rosnay, Joël De. 1997. « Y a-t-il un avenir après l'internet ? ». In Oudart, Le français dans le monde, Recherches et applications, Numéro spécial juillet.

Sfez, Lucien. 1992 [1988]. Critique de la communication, $2^{\mathrm{e}}$ éd. Paris : Seuil, Points Essais.

Smith, Steve \& Jacqueline Smith. 1998. Wordflo, Your Personal English Organizer. Londres : Addison Wesley Longman.

Varela, Francisco J. 1989. Connaître les sciences cognitives, tendances et perspectives. Paris: Seuil, traduit de (1988) Cognitive Science, A Cartography of Current Ideas.

Vignaud, J.-Claude. 1997. « Le professeur et ses élèves dans la démarche d'apprentissage ». Les langues modernes 2, 24-31.

Walski, Jennifer. 1993. « Du guidage en autonomie guidée ». Les langues modernes 93-1, 57-66.

Weil-Barais, Annick et al. 1993. L'homme cognitif. Paris : Presses universitaires de France.

\section{NOTES}

1. Cette introduction historico-étymologique est adaptée de l'article « Réseaux (philosophie de l'organisation) » de l'Encyclopcedia Universalis (1995).

2. Pour les différents niveaux d'apprentissage, voir G. Bateson (1964) cité par Carré et al. (1997 : 214-215).

3. D'après Dieuzeide (1994: 17), la didactique serait la gestion de l'information tandis que la pédagogie serait l'économie de la communication.

4. voir Laborit, 1974 : chapitre I.

5. Il est frappant de constater la similitude topographique entre le cortex cérébral tel qu'illustré par exemple chez Changeux (op. cit. Fig. 15, p. 63) et une carte du réseau Internet telle qu'on peut la voir à l'adresse <http://www.cs.bell-labs.com/ ches/map/gallery/isp-ss.gif>. 


\section{RÉSUMÉS}

Le thème proposé à la réflexion des congressistes du GERAS en mars 1999, «Les réseaux ", a été pour nous l'occasion de parcourir divers aspects de la métaphore réticulaire dans la perspective de notre travail de didacticien des langues. Nous évoquons le réseau comme filet permettant de retenir l'apprenant puis permettant à celui-ci de retenir le savoir ; puis le réseau comme support de l'information et du savoir, avec en particulier les réseaux sémantiques.

The theme of the March 1999 GERAS conference, "Networks" was for us an opportunity to explore various aspects of the metaphor of the network, in connection with our daily work as a language teacher. This article deals with the metaphor of the fishing-net used to capture first the learner's interest, then to help the learner retain knowledge; we then present the importance of semantic networks and mind-maps in the understanding and memorising of new items.

\section{INDEX}

Mots-clés : apprentissage, didactique des langues, Internet, mémorisation, motivation, réseau, réseau sémantique

Keywords : language acquisition, language learning, network, semantic network

\section{AUTEUR}

\section{JOSEPH RÉZEAU}

Joseph Rézeau enseigne l'anglais à l'université de Haute Bretagne Rennes 2, au département des Arts et en Didactique des langues. Il pratique l'enseignement assisté par ordinateur depuis le début des années 1980 et effectue actuellement une recherche sur la redéfinition de la médiation pédagogique en langues dans un environnement d'apprentissage multimédia.

joseph.rezeau@orange.fr 